\title{
Linearity and Power Characteristics of SiGe HBTs at High Temperatures for RF Applications
}

\author{
Kun-Ming Chen, Member, IEEE, An-Sam Peng, Guo-Wei Huang, Member, IEEE, Han-Yu Chen, Sheng-Yi Huang, \\ Chun-Yen Chang, Fellow, IEEE, Hua-Chou Tseng, Tsun-Lai Hsu, and Victor Liang
}

\begin{abstract}
In this paper, the power gain, power-added efficiency $(P A E)$ and linearity of power SiGe heterojunction-bipolar transistors at various temperatures have been presented. The power characteristics were measured using a two-tone load-pull system. For transistors biased with fixed base voltage, the small-signal power gain and $P A E$ of the devices increase with increasing temperature at low base voltages, while they decrease at high base voltages. Besides, the linearity is improved at high temperature for all voltage biases. However, for devices with fixed collector current, the small-signal power gain, $P A E$, and linearity are nearly unchanged with temperature. The temperature dependence of power and linearity characteristics can be understood by analyzing the cutoff frequency, the collector current, Kirk effect and nonlinearities of transconductance at different temperatures.
\end{abstract}

Index Terms-Linearity, power-added efficiency, power gain, SiGe HBT, temperature.

\section{INTRODUCTION}

$\mathbf{I}$ $\mathrm{N}$ RECENT years, the mobile communication market has grown rapidly. Future wireless communication systems will require higher speed, higher operation frequency, more functionality, light weigh, low power consumption, and low cost. One of the solutions to meet these requirements is to integrate receiver, transmitter, and other processing circuits on a single chip. Devices made from III-V materials exhibit high-speed and high-frequency operations but are rather difficult for highlevel integration. On the other hand, the conventional low-cost Si-based devices with poor frequency response are not suitable for microwave application. Fortunately, with recent technology advancements that push cutoff frequency and maximum oscillation frequency over $200 \mathrm{GHz}$ [1], [2], SiGe HBTs have improved the high-frequency performance of conventional $\mathrm{Si}$ technology. Due to the higher performance than Si devices and higher integration level than III-V devices, SiGe HBTs are suited ideally for large-volume manufacturing of RF transceiver systems at and beyond $2.4 \mathrm{GHz}$ [3], [4]. Recently, SiGe hetero-

Manuscript received August 30, 2004; revised December 16, 2004. This work was supported in part by the R.O.C.'s National Science Council under Contract NSC93-2215-E-492-006. The review of this paper was arranged by Editor M. Ostling.

K.-M. Chen, A.-S. Peng, and G.-W. Huang are with the National Nano Device Laboratories, Hsinchu 300, Taiwan, R.O.C.

H.-Y. Chen, S.-Y. Huang, and C.-Y. Chang are with the Department of Electronics Engineering, National Chiao-Tung University, Hsinchu 300, Taiwan, R.O.C.

H.-C. Tseng, T.-L. Hsu, and V. Liang are with the United Microelectronics Corporation, Hsinchu 300, Taiwan, R.O.C.

Digital Object Identifier 10.1109/TED.2005.850633 junction-bipolar transistors (HBTs) have attracted much attention for power amplifier application because of their excellent microwave power performance and high thermal conductivity. A challenge faced by SiGe-based power amplifier technologies is providing sufficient high-voltage immunity without compromising power performance. By optimizing the SiGe process, the microwave power applications of SiGe-based HBT under investigation and development have moved from L-, S-, and C-band operations [5], [6] to X-band operation [7].

Since the power devices are operated at high power densities, the device will suffer a high junction temperature due to self-heating effect. Besides, for high-temperature applications, the temperature behavior of devices needs to be investigated and well modeled for circuit design. Therefore, we are interested to know the temperature effect on power characteristics of transistors. For device modeling, the thermal effect is usually taken into account by adding a thermal network, and it can well simulate the power characteristics at different biases [8]. However, the experimental results for temperature effect on power and linearity characteristics of SiGe HBTs have not been presented. In the past, the majority of literatures on temperature effects deals mainly with the dc characteristics and/or high-frequency behavior [9]-[11], and seldom addresses the power characteristics [12]. In this paper, we investigate temperature effects on the power gain, power-added efficiency and intermodulation distortion of SiGe HBTs.

\section{EXPERIMENTS}

Multi-finger SiGe HBTs were fabricated in a $0.24 \mu \mathrm{m}$ highvoltage SiGe HBT process. The devices exhibit a dc current gain of up to 181 and the $\mathrm{BV}_{\mathrm{CEO}}$ is $5.3 \mathrm{~V}$. Typical cutoff frequency $\left(f_{T}\right)$ and maximum oscillation frequency $\left(f_{\max }\right)$ are about $23 \mathrm{GHz}$ and $40 \mathrm{GHz}$, respectively. The devices under test consist of four HBT cells; each cell has four emitter fingers with dimensions of $0.24 \mu \mathrm{m} \times 32 \mu \mathrm{m}$. These devices were measured in the common-emitter mode on wafer with Cascade microwave probes. Power characterization was performed using a load pull system, which consisted of HP85122A and ATN LP1 (power parameter extraction software), at various temperatures. The source impedance was conjugately matched to the input impedance for maximizing the power flowing into the device, while the load impedance was tuned for maximum output power at input power $P_{\text {in }}=-10 \mathrm{dBm}$ for each biasing point. The two-tone test was carried out at $2.4 \mathrm{GHz}$ with a 1-MHz separation between the two signals. 


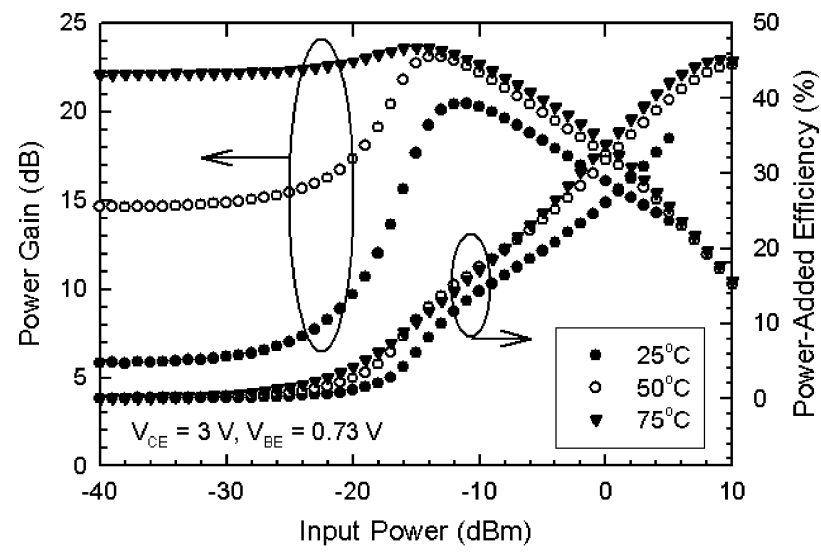

(a)

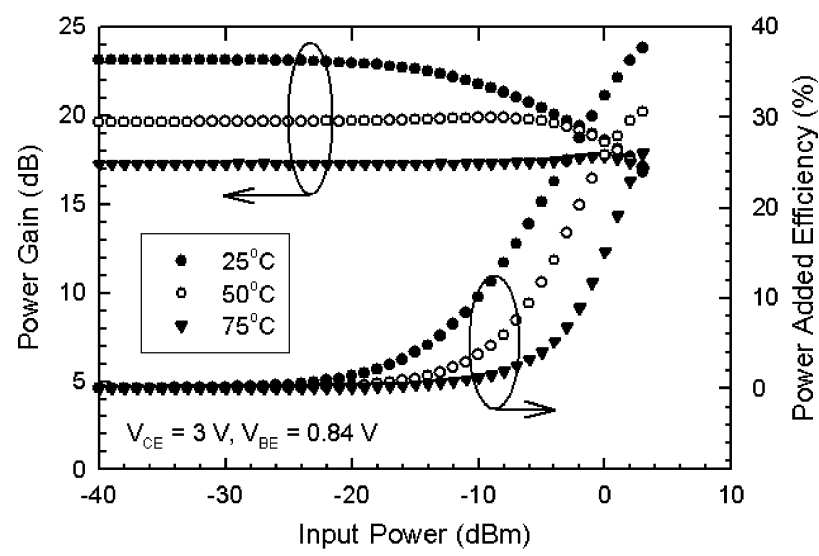

(b)

Fig. 1. Power gain and power-added efficiency versus input power for a SiGe $\mathrm{HBT}$ at (a) $V_{\mathrm{BE}}=0.73 \mathrm{~V}$ and (b) $V_{\mathrm{BE}}=0.84 \mathrm{~V}$ with different temperatures.

\section{RESULTS AND DISCUSSION}

\section{A. Power Gain}

Fig. 1 shows the power gain and power-added efficiency $(P A E)$ of a SiGe HBT at different biases and temperatures. At base voltage $V_{\mathrm{BE}}=0.73 \mathrm{~V}$, the power gain increases gradually with increasing input power level before gain compression. This phenomenon (i.e., gain expansion) is due to the exponential relation between the applied base voltage and collector current, and it takes place as device operates near cutoff region. When temperature rises, the power gain at small signal increases and the gain expansion will be suppressed and nearly disappear at $75^{\circ} \mathrm{C}$. It was observed and discussed in [12]. At $V_{\mathrm{BE}}=0.84 \mathrm{~V}$, the small-signal power gain $\left(G_{p}\right)$ decreases with increasing temperature, which is opposite to that at $V_{\mathrm{BE}}=0.73 \mathrm{~V}$. It indicates the temperature-dependent power characteristics will change with different bias conditions. Of course, the optimized source and load impedances have changed with temperature, however, the observation is similar to that with fixed impedances. For class-A operation, the small-signal power gain of amplifiers can be expressed as [13]

$$
G_{p}=\frac{f_{T}}{8 \pi\left(r_{E}+r_{B}+\pi f_{T} L_{E}\right) C_{\mathrm{BC}} f^{2}}
$$

where $r_{E}$ is the emitter resistance, $r_{B}$ is the base resistance, $L_{E}$ is the emitter lead inductance, $C_{\mathrm{BC}}$ is the base-collector capacitance, and $f$ is the operating frequency. In the above equa-

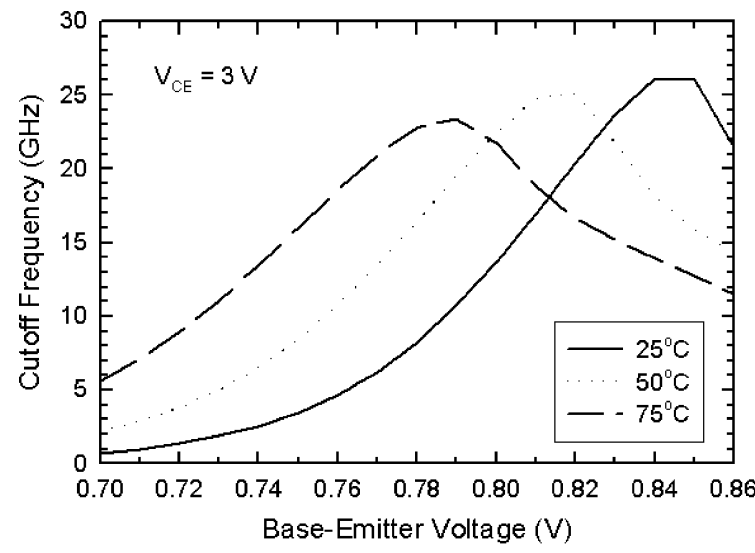

(a)

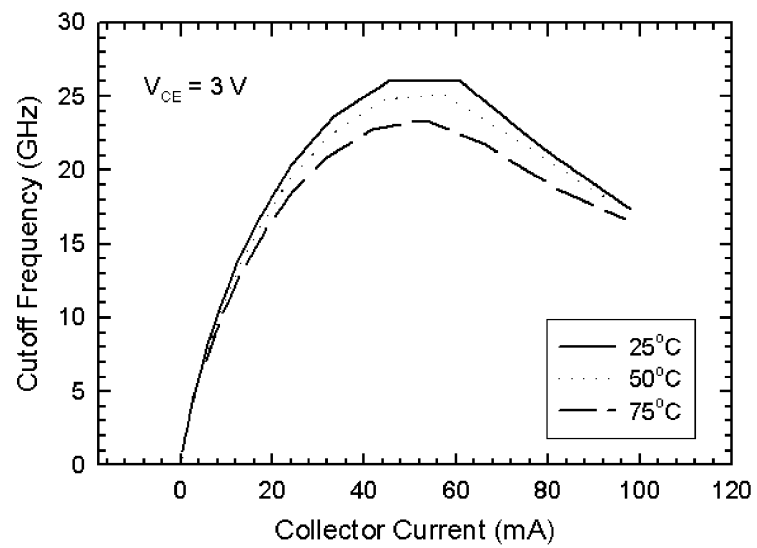

(b)

Fig. 2. Cutoff frequency (a) versus base-emitter voltage and (b) versus collector current at different temperatures.

tion, the cutoff frequency $\left(f_{T}\right)$ is the most temperature-sensitive parameters than the others. Hence we measured the cutoff frequency at different temperatures and the measured results are shown in Fig. 2. We found that the cutoff frequency increases with increasing temperature at low base voltage or low injection, while it decreases with increasing temperature at high injection. This observation can explain the temperature-dependent results in Fig. 1 by way of (1).

When we keep the base voltage with a constant, the increased temperature will increase the collector current and the base current due to the increase of intrinsic carrier concentration, as shown in Fig. 3. Because the depletion capacitance charging time is proportional to the inverse of the collector current, the increase of collector current with temperature will reduce the depletion capacitance charging time and thus increase the cutoff frequency in the low injection region. Hence, the small-signal power gain will increase with increasing temperature at $V_{\mathrm{BE}}=0.73 \mathrm{~V}$ [see Fig. 1(a)]. When the device operates in the high injection region, the Kirk effect will take place, in which the base region effectively extends into the collector. The extended base width leads to the fall-off of the cutoff frequency. As shown in Fig. 2(b), the critical current for the onset of Kirk effect is changed slightly with temperature, and the cutoff frequency decreases significantly with increasing current in high current region. Because the collector current will increase with increasing temperature at fixed $V_{\mathrm{BE}}$, the 


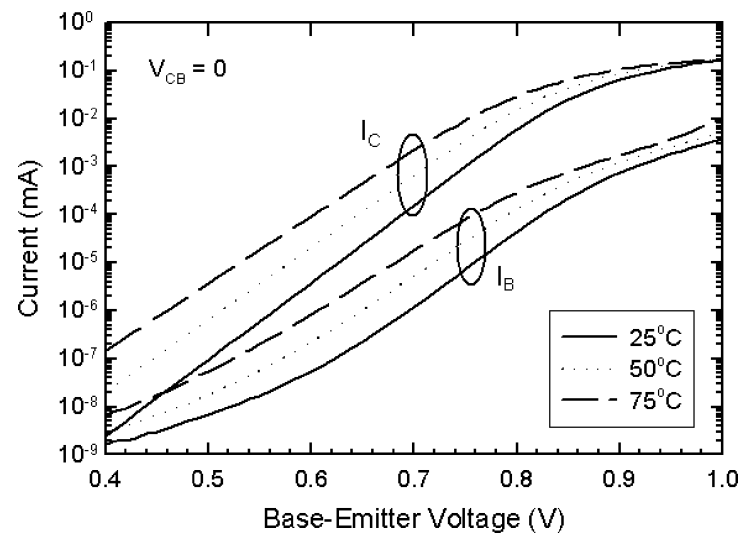

Fig. 3. Gummel plots of a SiGe HBT at different temperatures.

cutoff frequency will decrease with increasing temperature in high base voltage region. At $V_{\mathrm{BE}}=0.84 \mathrm{~V}$, Kirk effect has occurred below $50{ }^{\circ} \mathrm{C}$, hence the power gain decreases with increasing temperature [see Fig. 1(b)].

When the input signal increases to very large values, the power gain may be compressed. The power devices will produce more apparent nonlinear output waveform when input power beyond the gain compression. These mechanisms will limit the maximum acceptable input and output power of power amplifiers. When the dynamic load line exceeds the border of output I-V curve, the output waveform will be clipped. These clipping effects are the main reasons causing gain compression [14]. In our experiment, we found that the average collector current is unchanged with input power in low input power region, while the average current will increase in higher input power region. The increasing in average collector current means the negative duty cycle of output $\mathrm{I}-\mathrm{V}$ waveform is suffering cutoff clipping effect. When the temperature increases, the collector current will increase at fixed base voltage, making the dc bias point keep more away from the cutoff region. It allows a lager magnitude of output waveform. As a result, the power gain after compression point will shift to higher values as the temperature increases (see Fig. 1). Especially, for devices operate near cutoff region, the increase of power gain will be more obvious than that at high voltage biases.

Fig. 4 shows the small-signal power gain as a function of collector current $\left(I_{C}\right)$ with different temperatures. For power amplifier circuits, it is necessary to tune the source and load impedances to optimized condition at ambient temperature. Hence, we changed the optimized source and load impedances with different temperatures to compare the power characteristics of transistors in room temperature and high-temperature applications. It was observed that the $G_{p}$ is changed slightly with temperature when the measurements were carried out by keeping a constant collector current. This is due to the fact that the change of cutoff frequency with temperature at fixed collector current is not so significant as compared to that at fixed base voltage. Besides, from Fig. 4(a), the power gain decreases slightly with increasing temperature due to the reduction of maximum cutoff frequency (see Fig. 2), which is attributed to the mobility degradation. The result of Fig. 4(a) is important, since it suggests that the $G_{p}$ will not be degraded for high-temperature application when the power amplifier is biased at a constant collector current.

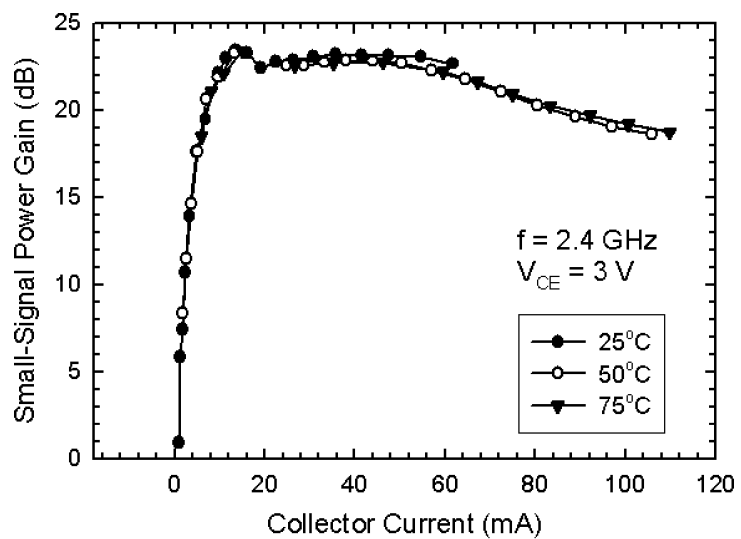

(a)

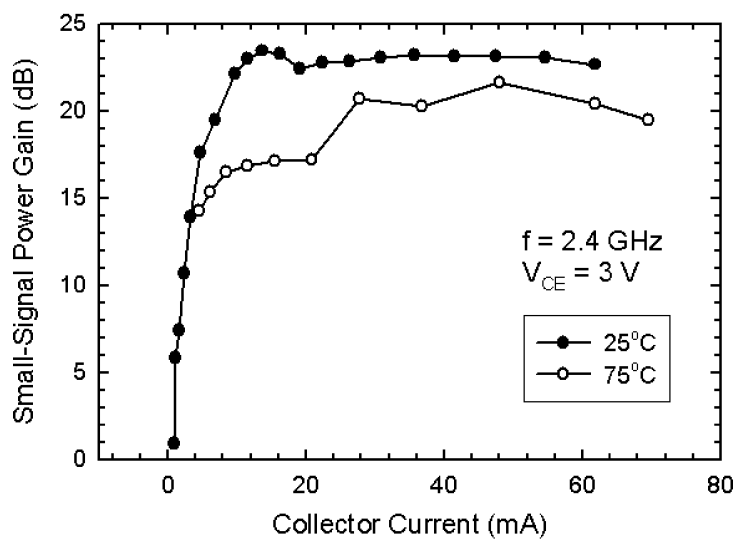

(b)

Fig. 4. Small-signal power gain versus collector current for a SiGe HBT at different temperatures. (a) Optimized source and load impedance were tuned for each temperature. (b) Optimized source and load impedances were tuned for $25^{\circ} \mathrm{C}$

When discussing the thermal stability of an amplifier circuit, the source and load impedances may be better to be kept at constant values for different temperatures due to the less change of impedances within small temperature range. The temperature dependence of power gain with fixed source and load impedances tuned at $25{ }^{\circ} \mathrm{C}$ is shown in Fig. 4(b). The power gain decreases with increasing temperature due to the mismatch of impedances at higher temperatures. Although the power gain has been reduced at higher temperatures, its deviation with temperature is still smaller than that with constant base voltage bias. It indicates that the $G_{p}$ of devices biased at constant collector current has higher immunity for temperature variation than that at constant base voltage.

\section{B. Power-Added Efficiency}

For power amplifiers used in mobile communication systems, high PAE is desirable. The power-added efficiency is defined as

$$
P A E=\frac{P_{\mathrm{out}}-P_{\mathrm{in}}}{P_{\mathrm{dc}}}=\eta_{C}\left(1-\frac{1}{G_{p}}\right)
$$

where $\eta_{C}$ is the collector efficiency and equals to $P_{\mathrm{out}} / P_{\mathrm{dc}}$. $P_{\mathrm{dc}}, P_{\mathrm{out}}$, and $P_{\mathrm{in}}$ are the dc power supply, ac output power, and ac input power, respectively. The temperature dependence of $P A E$ and collector efficiency is illustrated in Fig. 5. Because 


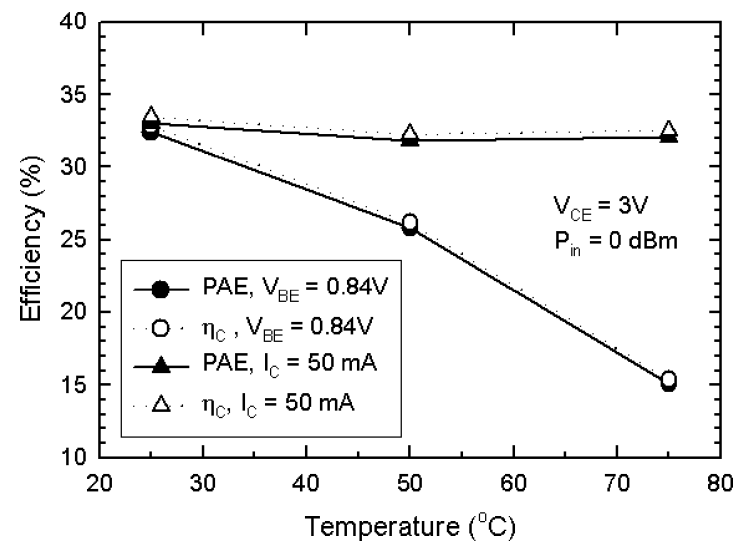

Fig. 5. Power-added efficiency and collector efficiency versus temperature at $V_{\mathrm{BE}}=0.84 \mathrm{~V}$ and $I_{C}=50 \mathrm{~mA}$.

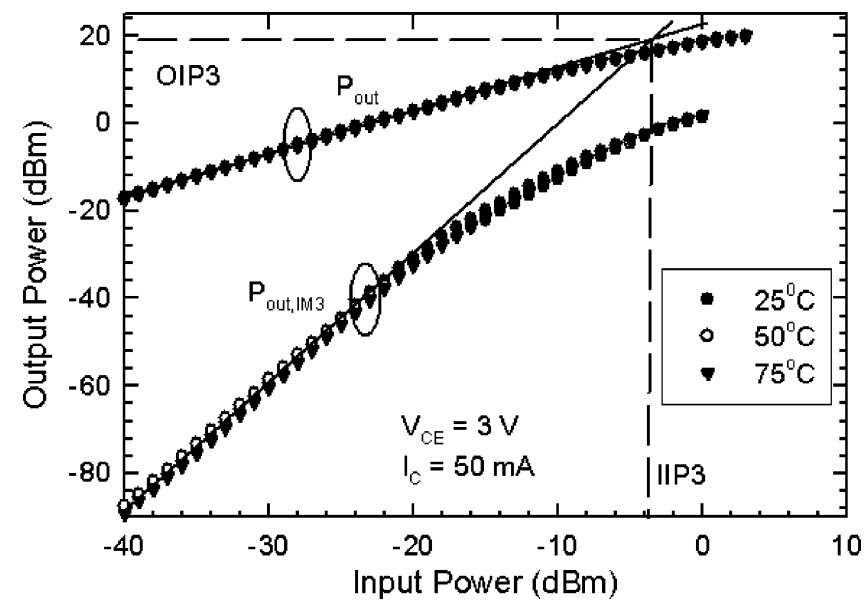

Fig. 6. Output power and third-order intermodulation power versus input power for a SiGe HBT at $I_{C}=50 \mathrm{~mA}$ with different temperatures.

of the high power gain in our devices, the collector efficiency is close to the PAE. In other words, the change of power gain due to temperature has little effect on the $P A E$, and only the change of $P_{\mathrm{dc}}$ and $P_{\text {out }}$ will affect the $P A E$ significantly.

At fixed base voltage $\left(V_{\mathrm{BE}}=0.84 \mathrm{~V}\right)$, the collector current increases with temperature, and thus the $P_{\mathrm{dc}}$ will increase. In addition, the output power is less dependent on temperature at $P_{\text {in }}=0 \mathrm{dBm}$. Therefore, the PAE has large amount of degradation at a high temperature. If the transistor is biased at constant collector current, i.e., constant dc power supply, as discussed in Section III-A, the power gain and output power is nearly unchanged with temperature. Therefore, the PAE will be slightly dependent on temperature as the collector current is kept to a constant.

\section{Linearity}

To study the temperature effects on the linearity of a $\mathrm{SiGe}$ HBT, the two-tone load-pull measurement was carried out. The most frequently used linearity figure-of-merit of a nonlinear microwave circuit is the third-order intercept point (IP3), at which the output power and third-order intermodulation (IM3) are equal, as shown in Fig. 6. For low distortion operation, the third-order intercept point should be as high as possible.

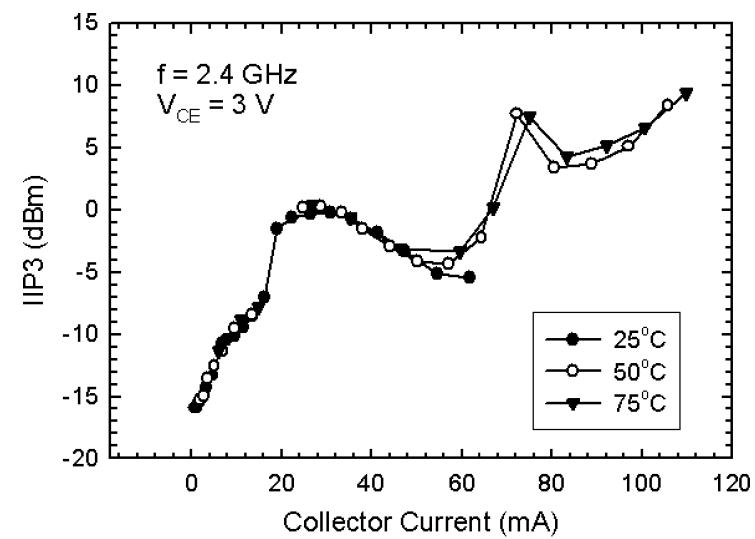

(a)

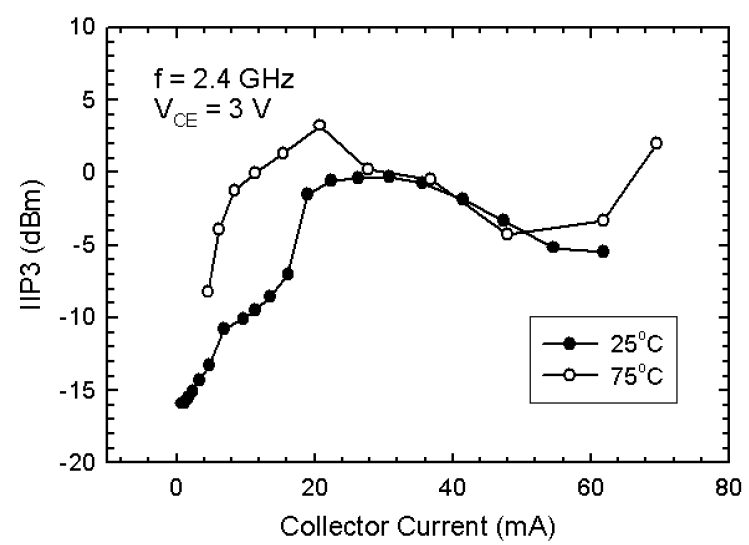

(b)

Fig. 7. IIP3 versus collector current for a SiGe HBT at different temperatures. (a) Optimized source and load impedances were tuned for each temperature. (b) Optimized source and load impedances were tuned for $25^{\circ} \mathrm{C}$.

The major nonlinear elements in a bipolar transistor are the collector current, base current, base-emitter charge, and base-collector charge. These nonlinear elements are dependent on temperature. Hence, the variation of temperature will change the device linearity. Fig. 7(a) shows the input IP3 (IIP3) as a function of collector current, where the optimized source and load impedances have been changed with temperature. It was observed that the IIP3 is nearly independent on the temperature and is only a function of collector current. The current dependence of IP3 for bipolar transistors has been widely discussed and analyzed by using Volterra series approach [16], [17] or large-signal compact model [18], [19]. As shown in Fig. 7(a), there are significant peaks and troughs in the IP3 behavior. Since the first trough of IIP3 curve roughly corresponds to the peak of cutoff frequency, as depicted in Figs. 7(a) and 2(b), the collector currents, which exceed the value at the trough, should suffer the Kirk effect. It is believed that in low current region, the distortion is dominated by the nonlinear contributions from transconductance $g_{m}$. As the current is increased, $g_{m}$ nonlinearities are decreased due to the increase of $g_{m}$ and the feedback effect of emitter and base resistances, and eventually, distortion from base-collector capacitance nonlinearities dominates. When the current increases to the vicinity of the first IP3 trough, distortion due to the Kirk effect begins to dominate. At the onset of the Kirk effect, the electron transit time, which is related to the diffusion charge, increases due to the 


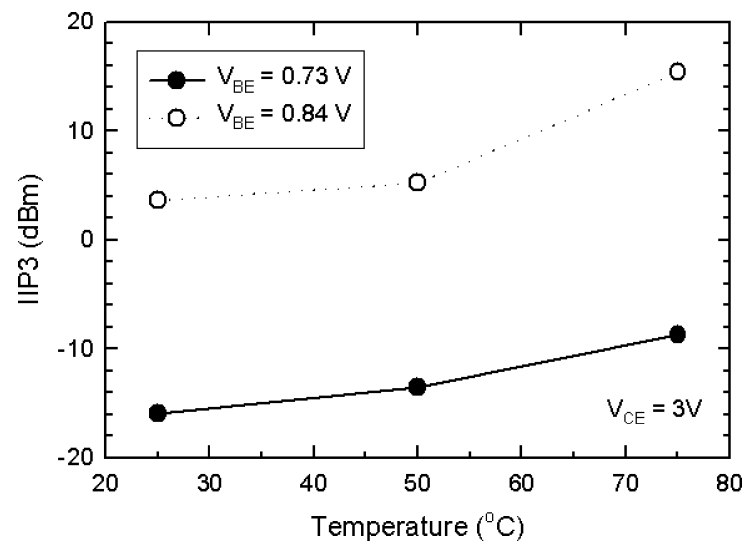

Fig. 8. IIP versus temperature for a SiGe $\mathrm{HBT}$ at fixed base voltages.

increase of the effective base width. In addition, the electrical field at the base-collector junction collapses and the width of the depletion region decreases, leading to an increased $C_{\mathrm{BC}}$ with a current dependence [18]. Therefore, the IP3 increases with current, and then levels off at highest currents [18]. Finally, the results in Fig. 7(a) also suggest that the linearity is less sensitive to temperature as the transistors are biased at constant collector currents.

The IIP3 as a function of collector current with different temperatures, where the optimized source and load impedances were tuned at $25{ }^{\circ} \mathrm{C}$ and kept at constant values for higher temperatures, is shown in Fig. 7(b). The IIP3 increases with increasing temperature at low current region, while it remains the same values at high current region. Although the load impedance has been mismatched from the maximum output power condition for higher temperatures, the IIP3 is still improved. It indicates the optimized load impedances for maximum output power and IP3 are not the same values.

Fig. 8 shows the temperature dependence of IIP3 for devices biased at fixed base voltages. The optimized source and load impedances were changed with temperature. At fixed $V_{\mathrm{BE}}$, the linearity will be improved at higher temperature for all biases. The observation is different from that in field-effect transistors [15], where the linearity degraded with temperature, indicating that SiGe HBTs will be a suitable alternative in high-temperature application if the linearity is the main demand. Because the IP3 is a strong function of collector current, the change of linearity with temperature at fixed base voltage can be explained simply by the change of collector current. At $V_{\mathrm{BE}}=0.73 \mathrm{~V}$, the collector currents are $1.92,4.68$, and $11.7 \mathrm{~mA}$, respectively, for temperature $T=25^{\circ} \mathrm{C}, 50{ }^{\circ} \mathrm{C}$, and $75^{\circ} \mathrm{C}$. These currents cover the range before the first peak of IIP3 curve, as shown in Fig. 7(a), so the distortion is dominated by the $g_{m}$ nonlinearity and improves as current is increased. Hence, the linearity will be improved with temperature at low base voltage. When the base voltage increases to $0.84 \mathrm{~V}$, the collector currents are $47.5,88.3$, and $146 \mathrm{~mA}$, respectively, for $T=25^{\circ} \mathrm{C}, 50{ }^{\circ} \mathrm{C}$, and $75^{\circ} \mathrm{C}$. The distortion is dominated by the nonlinearity from Kirk effect. The IP3 is also increased with collector current. Consequently, the linearity will also be improved with increasing temperature for high bias conditions.

\section{CONCLUSION}

Linearity and power characteristics of SiGe HBTs at various operation temperatures have been analyzed. The small-signal power gain and $P A E$ of SiGe HBTs increase with temperature at low base voltages, when we keep the base voltage with a constant value. Because the collector current increases with temperature, the cutoff frequency will increase, leading to the increase of power gain. However, the small-signal power gain and $P A E$ decrease with temperature at high base voltages, due to the decrease of the cutoff frequency. Moreover, we found that the linearity at fixed base voltage increases with temperature for all biases. It is attributed to the temperature variation of collector current, which affects the nonlinearities of transconductance, electron transit time and base-collector capacitance. In addition, when we keep the collector current with a constant, the power gain, $P A E$, and linearity are nearly independent on the temperature. It is therefore suggested that the $\mathrm{Si} / \mathrm{SiGe}$ amplifier performance can be made more stable to the change of temperature by biasing the HBT at a constant current. On the other hand, for high-temperature applications, the amplifier can be biased at lower base voltages to obtain higher power gain and linearity, as well as lower dc power consumption.

\section{ACKNOWLEDGMENT}

The authors would like to thank the staff of the United Microelectronics Corporation for their helpful comments and the staff of Radio Frequency Technology Center in NDL for measurement support.

\section{REFERENCES}

[1] J.-R. Rieh, B. Jagannathan, H. Chen, K. T. Schonenberg, D. Angell, A. Chinthakindi, J. Florkey, F. Golan, D. Greengerg, S.-J. Jeng, M. Khater, F. Pagette, C. Schnabel, P. Smith, A. Stricker, K. Vaed, R. Volant, D. Ahlgren, G. Freenman, K. Stein, and S. Subbanna, "SiGe HBT's with cut-off frequency of $350 \mathrm{GHz}$," in IEDM Tech. Dig., 2002, pp. 771-774.

[2] K. Washio, "SiGe HBT and BiCMOS technologies," in IEDM Tech. Dig., 2003, pp. 113-116.

[3] M. Case, S. A. Maas, L. Larson, D. Rensch, D. Harame, and B. Meyerson, "An X-band monolithic active mixer in SiGe HBT technology," in IEEE MTT-S Dig., 1996, pp. 655-658.

[4] J. S. Rieh, L. H. Lu, L. P. B. Katehi, P. Bhattacharya, E. T. Croke, G. E. Ponchak, and S. A. Alterovitz, "X- and Ku-band amplifiers based on Si/SiGe HBT's and micromachined lumped components," IEEE Trans. Microw. Theory Tech., vol. 46, no. 5, pp. 685-694, May 1998.

[5] J. N. Burghartz, J.-O. Plouchart, K. A. Jenkins, C. S. Webster, and M. Soyuer, "SiGe power HBT's for low-voltage, high performance RF applications," IEEE Electron. Device Lett., vol. 19, no. 4, pp. 103-105, Apr. 1998.

[6] U. Erben, M. Wahl, A. Schuppen, and H. Schumacher, "Class-A SiGe HBT power amplifiers at C-band frequencies," IEEE Microw. Guided Wave Lett., vol. 5, no. 12, pp. 435-436, Dec. 1995.

[7] M. Zhenqiang, S. Mohammadi, P. Bhattacharya, L. P. B. Katehi, S. A. Alterovitz, and G. E. Ponchak, "A high-power and high-gain X-band $\mathrm{Si} / \mathrm{SiGe} / \mathrm{Si}$ heterojunction bipolar transistor," IEEE Trans. Microw. Theory Tech., vol. 50, no. 4, pp. 1101-1108, Apr. 2002.

[8] B. Schaefer and M. Dunn, "Pulsed measurements and modeling for electro-thermal effects," in Proc. IEEE BCTM, 1996, pp. 110-117.

[9] E. J. Prinz and J. C. Sturn, "Base transport in near-ideal graded-base $\mathrm{Si} / \mathrm{Si}_{1-\mathrm{x}} \mathrm{Ge}_{\mathrm{x}} / \mathrm{Si}$ heterojunction bipolar transistors from $150 \mathrm{~K}$ to 370 K," in IEDM Tech. Dig., 1990, pp. 975-978.

[10] J. D. Cressler, E. F. Crabbe, J. H. J. H. Comfort, J. Warnock, K. A. Jenkins, J. M. C. Stork, and J. Y.-C. Sun, "Profile scaling constraints for ion-implanted and epitaxial bipolar technology designed for $77 \mathrm{~K}$ operation," in IEDM Tech. Dig., 1991, pp. 861-864. 
[11] E. F. Crabbe, G. L. Patton, J. M. C. Stork, J. H. Comfort, B. S. Meyerson, and J. Y.-C. Sun, "Low temperature operation of Si and SiGe bipolar transistors," in IEDM Tech. Dig., 1990, pp. 17-20.

[12] A. S. Peng, K. M. Chen, G. W. Huang, M. H. Cho, S. C. Wang, Y. M. Deng, H. C. Tseng, and T. L. Hsu, "Temperature effect on power characteristics of SiGe HBTs," in IEEE MTT-S Dig., 2004, pp. 1955-1958.

[13] G. B. Gao, H. Morkoc, and M. C. Frank, "Heterojunction bipolar transistor design for power applications," IEEE Trans. Electron Devices, vol. 39, no. 9, pp. 1987-1997, Sep. 1992.

[14] M. Y. Frankel and D. Pavlidis, "Large-signal modeling and study of power saturation mechanisms in heterojunction bipolar transistors," in IEEE MTT-S Dig., 1991, pp. 127-130.

[15] A. Ahmed, S. S. Islam, and A. F. M. Anwar, "Temperature dependence of intermodulation and linearity in GaN based devices," in IEEE RFIC Symp. Dig., 2001, pp. 275-278.

[16] M. Vaidyanathan, M. Iwamoto, L. E. Larson, P. S. Gudem, and P. M. Asbeck, "A theory of high-frequency distortion in bipolar transistors," IEEE Trans. Microw. Theory Tech., vol. 51, no. 2, pp. 448-460, Feb. 2003.

[17] G. Niu, Q. Liang, J. D. Cressler, C. S. Webster, and D. L. Harame, "RF linearity characteristics of SiGe HBTs," IEEE Trans. Microw. Theory Tech., vol. 49, no. 9, pp. 1558-1565, Sep. 2001.

[18] M. Iwamoto, P. M. Asbeck, T. S. Low, C. P. Hutchinson, J. B. Scott, A. Cognata, Q. Xiaohui, L. H. Camnitz, and D. C. D'Avanzo, "Linearity characteristics of GaAs HBT's and the influence of collector design," IEEE Trans. Microw. Theory Tech., vol. 48, no. 12, pp. 2377-2388, Dec. 2000.

[19] X. Yuan, D. Y. C. Lie, L. E. Larson, J. Blonski, J. Gross, M. Kumar, J. Mecke, A. Senior, Y. Chen, A. Poh, and D. Harame, "RF linearity study of SiGe HBT's for low power RFIC design, Part I," in Proc. 3rd Int. Conf. Microwave Millmeter Wave Technology, 2002, pp. 70-73.

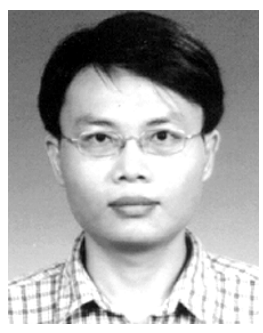

Kun-Ming Chen (M'01) received the M.S. and $\mathrm{Ph} . \mathrm{D}$. degrees in electronics engineering from National Chiao-Tung University, Hsinchu, Taiwan, R.O.C., in 1996 and 2000, respectively.

He joined the National Nano Device Laboratories, Hsinchu, in 2000 as an Associate Researcher. He was engaged in research on the microwave device process and characterization

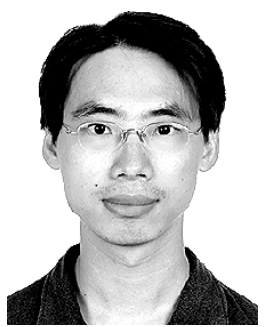

An-Sam Peng was born in Hsinchu, Taiwan, R.O.C., in 1976. He received the B.S. degree in electronics engineering from Feng-Chia University in 1999 and the M.S. degree in electrical engineering from $\mathrm{Na}-$ tional Chung Hsing University in 2001.

He joined the National Nano Device Laboratories, Hsinchu, in 2002 as an Assistant Researcher. His research interests focus on power characterization of SiGe HBTs, passive device models, and RFIC process design kit.

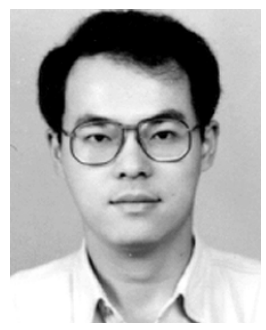

Guo-Wei Huang (S'94-M'97) was born in Taipei, Taiwan, R.O.C., in 1969. He received the B.S. degree in electronics engineering and the Ph.D. degree from National Chiao-Tung University, Hsinchu, Taiwan, in 1991 and 1997, respectively.

He joined National Nano Device Laboratories, Hsinchu, Taiwan, in 1997 as an Associate Researcher. His current research interests focus on microwave device design, characterization, and modeling.

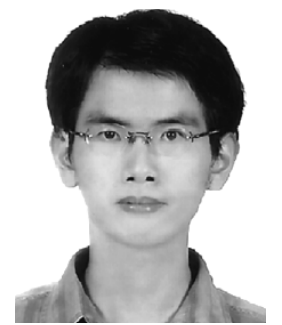

Han-Yu Chen received the B.S. degrees in electrical engineering from National Sun Yet-Sen University, Kaohsiung, Taiwan, R.O.C., in 2000. He is currently pursuing the Ph.D. degree in the Electronics Engineering Department of National Chiao-Tung University, Hsinchu, Taiwan.

His research interests include microwave device process and characterization.

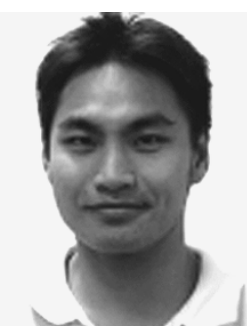

Sheng-Yi Huang was born in Yunlin, Taiwan, R.O.C., in 1978. He received the B.S. degree in electrical engineering from National Cheng Kung University, Tainan, Taiwan, in 2001 and the M.S.E.E. degree in 2003 from the National Chiao Tung University (NCTU), Hsinchu, Taiwan, where he is currently pursuing the Ph.D. degree in electronic engineering.

He joined the Mixed-Signal Department of the United Microelectronics Corporation, Hsinchu, in August 2003. His current research focuses on RF CMOS/BiCMOS device issues, including modeling, noise characteristics, and reliability.

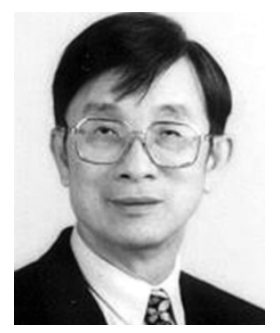

Chun-Yen Chang (F'88) was born in Feng-Shan, Taiwan, R.O.C. He received the B.S. degree in electrical engineering from Cheng Kung University, Tainan, Taiwan, in 1960, the M.S. degree in tunneling in semiconductor-supercondutor junctions, and the $\mathrm{Ph} . \mathrm{D}$. degree in carrier transport across metal-semiconductor barriers, both from National Chiao-Tung University (NCTU), Hsinchu, Taiwan, in 1969 .

He has devoted himself to education and academic research for more than 40 years. He has contributed profoundly to the areas of microelectronics and optoelectronics, including the invention of the method of low-pressure-MOCVD-using tri-ethyl-gallium to fabricate light-emitting diode laser, and microwave transistors, Zn-incorporation of $\mathrm{SiO}$ for stabilization of power devices, and nitridation of $\mathrm{SiO}$ for ULSIs, etc. In 1963, he joined NCTU to serve as an Instructor establishing a high vacuum laboratory. In 1964, he and his colleague established the Semiconductor Research Center (SRC) at NCTU with a very up-to-date, albeit homemade, facility for silicon device processing, where they made the nation's first Si Planar transistor in April 1965, and subsequently the first IC in August 1966. In 1968, he published Taiwan's first-ever semiconductor paper in the international journal Solid State Electronics. In 1969, he became a Full Professor, teaching solid-state physics, quantum mechanics, semiconductor devices, and technologies. From 1977 through 1987, he single-handedly established a strong electrical engineering and computer science program at NCKU where GaAs, $\alpha$-Si, poly-Si researches were established in Taiwan for the first time. Since 1987, he served consecutively as Dean of Research (1987-1990), Dean of Engineering (1990-1994), and Dean of Electrical Engineering and Computer Science (1994-1995). Simultaneously, he was serving as the Founding President of National Nano Device Laboratories (NDL) from 1990 through 1997. In 1997, he became Director of the Microelectronics and Information System Research Center (MIRC), NCTU (1997-1998). Many of his former students have since become founders of the most influential hi-tech enterprises in Taiwan, namely the United Microelectronics Corporation, TSMC, Winbond, MOSEL, Acer, Leo, etc. In August 1998, he was appointed President of NCTU. As the National-Chair-Professor and President of NCTU, his vision is to lead the university for excellence in engineering, humanity, art, science, management, and bio-technology. To strive torward a world class multidisciplinary university is the main goal to which he and his colleagues have committed.

Dr. Chang received the IEEE Third Millennium Medal in 2000. He is a member of Academia Sinica and a Foreign Associate of the National Academy of Engineering. 


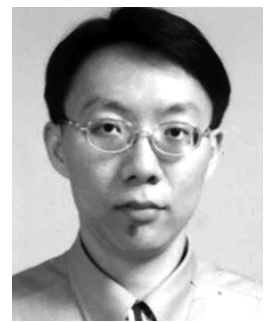

Hua-Chou Tseng received the B.S. and the Ph.D. degrees in electronic engineering from National ChiaoTung University, Taiwan, R.O.C., in 1991 and 1997, respectively. His Ph.D. research included low-temperature $\mathrm{Si} / \mathrm{SiGe}$ selective epitaxial growth and gas source doping for ultra-shallow junction formation.

From 1997 to 2000, he was with the United Microelectronics Corporation (UMC), Hsinchu, Taiwan, helping to develop the low-power 6T-SRAM, including the process integration and the device tuning of $0.25-\mu \mathrm{m}, 0.18-\mu \mathrm{m}$, and $0.15 \mu \mathrm{m}$ generations. He is currently the Group Leader of RF-CMOS and SiGe-BiCMOS technology development at UMC.

Dr. Tseng an international technical committee member of the International SiGe Technology and Device Meeting (ISTDM).

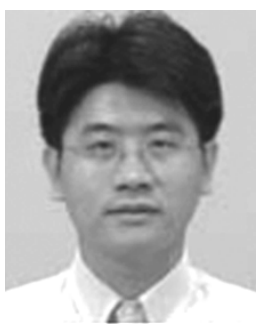

Tsun-Lai Hsu was born in Chi-I, Taiwan, R.O.C., in 1968. He received the B.S. degree in electric engineering from National Taiwan Ocean University in 1990 and the M.S. and Ph.D. degrees in electrical engineering from National Tsing-Hua University, Hsinchu, Taiwan, in 1992 and 1996, respectively. His Ph.D. research included reliability study, base resistance physical analysis, and $1 / f$ noise measurement for poly-emitter BJT.

He joined ERSO/ITRI to develop the RF doublepoly BJT technology, including RF/noise characteristics measurement and analysis. He worked on $0.18-\mu \mathrm{m} \mathrm{SiGe-BiCMOS}$ technology development at United Microelectronics Corporation (UMC), Hsinchu, in 2001 and 2002. Since 2002, he has been working in RF passive/active devices design for RF-CMOS at UMC. His current research is focused on inductors, substrate noise isolation, and RF deembedding methodology designs.

Victor Liang, photograph and biography not available at the time of publication. 